\title{
Circulating Tumour DNA as a Biomarker Source in Metastatic Prostate Cancer
}

\author{
Gillian Vandekerkhove, Alexander W. Wyatt ${ }^{凶}$ \\ Vancouver Prostate Centre, Department of Urologic Sciences, University of British Columbia, Vancouver, Canada
}

\begin{abstract}
Tumour molecular features are increasingly linked to treatment response and patient prognosis in advanced prostate cancer. Plasma cell-free circulating tumour DNA (ctDNA) isolated from a minimally invasive blood draw offers a convenient source of tumour material to develop clinical biomarkers. Importantly, the burden of ctDNA in the blood has strong prognostic implications at different points during the natural history of metastatic progression. In prostate cancer, the identification of somatic profiles from ctDNA requires a broad next-generation sequencing approach because of the low mutation rate and frequent structural rearrangements. Nevertheless, comparison of genomic profiles between liquid and tissue biopsies has demonstrated that ctDNA is a surrogate for tumour tissue in the metastatic setting. Our understanding of resistance to androgen receptor (AR) directed therapies has been significantly augmented by the frequent detection of $A R$ gene amplifications, mutations, and structural rearrangements via liquid biopsy. Furthermore, early studies suggest that distinct molecular subtypes derived from ctDNA profiling can help determine the optimal therapeutic regimen for an individual patient and enable real-time monitoring for therapy response and resistance. Indeed, in clinical trials targeting the DNA damage repair pathway in prostate cancer, ctDNA-based assessment of DNA repair status is already under evaluation as a predictive biomarker. Recent advances in the study of circulating DNA fragments now make it possible to interrogate aspects of the epigenome. In this review, we describe the various applications of plasma ctDNA in metastatic prostate cancer, including its potential role as a clinically informative liquid biomarker.
\end{abstract}

\section{Etiology of Cell-Free DNA}

Upon cell death, genomic DNA fragments can diffuse into sur-rounding bodily fluids. The most familiar source of cellfree DNA (cfDNA) is peripheral blood, but it can also be purified from urine, sputum, cerebrospinal fluid, and ascites. In healthy individuals, the cfDNA in each fluid is derived from cell types in the immediate neighbourhood. Therefore, in blood plasma, most cfDNA originates from hematopoietic cells $[1,2]$. With conventional extraction methodology, blood from healthy individuals yields about five nanograms of cfDNA (approximately 750 diploid genomes) per milliliter of double-spun plasma [3]. Plasma cfDNA has a periodic 167 base pair fragment pattern consistent with apoptotic processing, representing the intervals at which caspase-activated DNase cleaves DNA [4]. The presence of high molecular weight DNA in processed plasma is indicative of pre-analytic failure; proper collection, storage, and processing of plasma cfDNA is crucial to downstream success [5].

Injury and disease can alter the etiology of cfDNA [1,6,7]. In cancer patients, genomes from tumour cells undergoing apoptosis can be shed into body fluids. These tumour-derived cfDNA fragments are termed circulating tumour DNA (ctDNA), and they can be detected against a backdrop of cfDNA from benign cells using assays to identify somatic alterations or epigenetic marks. The half-life of cfDNA is typically measured in hours but varies by the enzymatic activity in each body fluid [8]. Moreover, the kidneys, liver, and spleen all clear cfDNA fragments in circulating blood. This rapid turnover means that detection of ctDNA in blood represents a real-time cancer biopsy.

\section{Key Words}

Circulating tumour DNA, cell-free nucleic acids, prostatic neoplasms, castrationresistant, liquid biopsy, recombinational DNA repair, DNA mismatch repair, androgen antagonists, biomarkers

\section{Competing Interests}

Alexander Wyatt reports personal fees from AstraZeneca, grants and personal fees from Jannsen, personal fees from Bayer, outside the submitted work. Gillian Vandekerkhove reports no competing interests.

\section{Article Information}

Received on June 25, 2020

Accepted on September 8, 2020

Soc Int Urol J. 2020;1(1):39-48 


\section{Abbreviations \\ $A R$-GSRs androgen receptor genomic structural rearrangements \\ cfDNA cell-free DNA \\ ctDNA circulating tumour DNA \\ HRR homologous recombination repair \\ mCRPC metastatic castration-resistant prostate cancer \\ MMRd mismatch repair deficiency \\ PARP poly (ADP-ribose) polymerase \\ $\mathrm{PCa} \quad$ prostate cancer \\ WGS whole-genome sequencing}

\section{Approaches for Cell-Free Circulating Tumour DNA Analysis}

In cancer patients, the proportion of cfDNA that is tumour-derived (ie, the ctDNA fraction) is highly variable [9]. This variability cannot be easily estimated without profiling purified cfDNA, and represents the major technical challenge for ctDNA detection and characterization. Assays must be highly sensitive to detect the possibility of rare ctDNA fragments diluted in hundreds to thousands of normal cfDNA fragments [10].

Polymerase chain reaction-based approaches to detect or characterize ctDNA rely on either prior knowledge of tumour genotype (eg, from whole-exome sequencing of the matched primary tumour) or the plausible presence of recurrent hotspot mutations. Unfortunately, with the possible exceptions of the $A R$ and SPOP genes, prostate cancer $(\mathrm{PCa})$ does not harbour highly recurrent hotspot mutations with obvious clinical relevance [11,12], thus broader approaches are required to capture the frequent genomic structural rearrangements and copy number changes [13].

At present, most clinical research using ctDNA in PCa has applied targeted next-generation sequencing approaches that capture a limited number of exons for a set of cancer-related genes [14]. Because of the lower cost, targeted sequencing is preferable to conventional whole-exome or genome approaches. For tumour tissuebased analysis, sequencing coverage of $30 \mathrm{x}$ to $100 \mathrm{x}$ is adequate to characterize the somatic genome $[15,16]$. However, ideal sequencing depths for ctDNA are typically above 1000x and often considerably higher [10]. This can be expensive, and hence the selection of genes or regions for cfDNA sequencing is a delicate balance between cost, genome coverage, and desired detection sensitivity. Most commercial assays cover several ubiquitous cancer genes that are relevant for $\mathrm{PCa}$, such as TP53, MYC, and BRCA2. However, important PCa genes that are not always present in historical pan-cancer approaches include SPOP, FOXA1, and CDK12 [13]. The selection of regions to sequence within each gene must also be carefully considered; inclusion of intronic and untranslated regions can enable identification of structural rearrangements and gene deletions. In PCa, PTEN, RB1, MSH2, FOXA1, and AR are often disrupted by structural rearrangements affecting introns $[15,17-$ 20]. Some of these genes can also be perturbed by partial or entire locus deletions. From an assay design perspective, comprehensive detection of structural rearrangement breakpoints requires probes tiled across introns. Inclusion of introns (often spanning kilobases) in targeted sequencing assays can significantly increase the cost of ctDNA profiling.

The ctDNA fraction of a sample determines the type of somatic alterations that can be detected. Advances in library preparation techniques (eg, duplex unique molecular identifiers) and bioinformatic approaches (eg, digital error suppression) enable somatic mutation identification at $\sim 0.1 \%$ frequency $[21,22]$. However, mathematical limits regarding the detection of copy number changes cannot be easily overcome by technological improvements. For example, the detection of entire chromosome arm deletions typically requires a ctDNA fraction of at least 5\% (rare outside of progressing metastatic disease) when using reasonably cost-effective targeted sequencing approaches [23]. CtDNA purity must be even higher to enable detection of focal deletions. In $\mathrm{PCa}$, several focal copy number changes have clear clinical relevance (eg, deletions affecting PTEN, MSH2, $B R C A 2$ ) [24]. Therefore, cfDNA assays should report ctDNA fraction and discriminate between a true negative result (ie, tumour wild type status) and the inability to detect a change due to low tumour DNA purity.

In research settings, $\mathrm{PCa}$ cfDNA samples have been subjected to whole-exome or wholegenome sequencing $[18,23,25,26]$. Whole-exome sequencing is generally cost-justifiable only in samples with ctDNA fractions above $20 \%$ to $40 \%$, but in such scenarios can provide a snapshot of somatic mutations and copy number changes. Deep whole-genome sequencing (WGS) of cfDNA is not feasible outside bespoke analyses, but low-pass WGS is a cost-effective alternative that has shown promise for wide uptake [27]. With this method, the entire genome is sequenced at a shallow depth, normally less than 1x. Low-pass WGS can provide an estimate of ctDNA fraction (although typically not below $3 \%$ to $5 \%$ ) and yields a low resolution genome-wide copy number profile. Since no targeted capture steps are required, it is cheap and quick to perform, and software packages for data analysis are publicly available [27]. However, low-pass WGS does not inform on somatic mutations, complex structural rearrangements, or focal copy number changes. Furthermore, the continual improvement of modular capture assays and targeted designs incorporating genome-wide targets means 
that it is possible to incorporate a backbone for wholegenome copy number profiling (eg, leveraging germline single nucleotide polymorphisms) into modern targeted sequencing assays.

Ideally, deep sequencing of plasma cfDNA is accompanied by sequencing of matched leukocytes as a germline surrogate. In addition to identifying pathogenic germline alterations affecting genes such as BRCA2 and TP53 [28], leukocyte sequencing also allows resolution of somatic mutations in cfDNA related to clonal hematopoiesis of indeterminate potential rather than cancer [17,29].

In summation, these factors significantly impact the detection sensitivity of each cfDNA profiling approach, and can lead to divergence in the results when comparing commercials tests with one another or with research assays $[13,30,31]$. Mutations with low variant allele fractions are particularly unreliable, which is likely due to clonal hematopoiesis and somatic expansions. End users must be aware of the limits of detection for their chosen assay. Ultimately, there is no single ctDNA testing approach that can inform on all possible scenarios in prostate cancer, and therefore, the choice should be governed by the scientific or clinical question of the investigator.

\section{Circulating Tumour DNA Abundance as a Prognostic Biomarker}

The abundance of ctDNA is a potentially clinically impactful variable, even without further characterization of molecular subtype. In metastatic castration-resistant prostate cancer (mCRPC), plasma ctDNA abundance is associated with clinical measures of disease burden such as prostate-specific antigen level and the presence of visceral metastatic lesions [23,26,3234]. Accordingly, high ctDNA fractions are associated with poor overall survival and short progression-free survival in $\mathrm{mCRPC}$ patients treated with standard of care $[23,32,35-37]$. The converse is also true: low or undetectable ctDNA appears to be a marker of good prognosis [23]. Importantly, ctDNA fraction in mCRPC appears to provide independent prognostic information to standard clinical factors, suggesting that assays of ctDNA abundance could become part of prognostic models $[23,37]$.

Since ctDNA abundance is closely related to the volume of proliferative disease, effective therapy has a rapid impact, and therefore blood collection before treatment or at clinical progression is recommended to maximize the chance of sufficient ctDNA for genomic characterization $[17,37,38]$. In metastatic castrationsensitive $\mathrm{PCa}$, one week of androgen-deprivation therapy can reduce ctDNA fractions by 10 -fold [38]. In mCRPC, declines in ctDNA are associated with prostate-specific antigen responses to abiraterone or enzalutamide, and patients with a rising ctDNA fraction while on treatment are at greater risk of progression [26,39-41]. The detection of changes in ctDNA fraction during treatment is a potential surrogate biomarker of response and should be explored in prospective biomarker trials.

\section{Relationship of Circulating Tumour DNA to Tumour Tissue Biopsy}

Tumour molecular features derived from liquid biopsies are typically expected to align with those from tissuebased analyses. In a study of 45 patients with mCRPC, deep targeted sequencing of same-day metastatic tissue biopsies and plasma cfDNA collections demonstrated high concordance for typical $\mathrm{PCa}$ driver gene alterations such as TP53 mutation, $A R$ amplification, SPOP mutation, and PTEN deletion [42]. In a parallel study, copy number profiles were highly concordant when applying low-pass WGS to mCRPC patientmatched tissue and ctDNA [43]. More recently, high tissue-ctDNA concordance for driver gene alterations has been reported in de novo metastatic castratesensitive PCa [38], and even among genomically or pathologically distinct patient subsets such as those with somatic mismatch repair defects or neuroendocrine features $[18,25]$. Collectively, the similarity between patient-matched tissue and ctDNA is consistent with the findings from rapid autopsy studies in which the vast majority of truncal driver alterations were conserved across metastatic sites $[44,45]$. Nevertheless, subclonal or late-arising alterations associated with acquired treatment resistance (eg, $A R$ amplification or mutation), and neutral passenger mutations, are likely to vary between metastatic lesions and therefore between a single biopsy site and ctDNA.

\section{DNA Damage Repair Defects as Prognostic and Predictive Biomarkers}

DNA damage repair defects are common in PCa, particularly in metastatic disease $[46,47]$, but their precise prognostic relevance is contingent on a number of factors. For example, there are several distinct DNA repair pathways and hundreds of individual genes with different degrees of involvement. Alterations in each pathway and even gene can have drastically different downstream genomic and clinical effects in addition to the specific class of alteration observed.

The most commonly affected DNA repair gene in $\mathrm{mCRPC}$ is BRCA2, which is altered at the germline and/or somatic level in $\sim 10 \%$ of patients $[15,48,49]$. Biallelic BRCA2 defects result in compromised ability to repair double-strand DNA breaks and reliance on 
alternative repair pathways that are more error-prone in this context [50]. Since monoallelic BRCA2 deletion is common in PCa, allelic status is key to reporting pathogenicity. In mCRPC with high ctDNA fractions, loss of heterozygosity across deleterious germline $B R C A 2$ mutations is evident in ctDNA, suggesting that cfDNA sequencing could identify patients with functional BRCA2 loss [51]. It is plausible that broad cfDNA sequencing will also be able to identify genomic signatures of defective homologous recombination repair (HRR), as has been demonstrated by tumour tissue sequencing $[52,53]$. Prospective and retrospective studies have suggested that BRCA2 defects detected via leukocyte and cfDNA sequencing associate with poor mCRPC outcomes in the context of AR-targeted therapy $[23,37,51,54]$. An association between plasma ctDNA DNA repair defects and poor outcomes has also been observed in metastatic castrationsensitive disease [37]. However, these associations appear variable across patient cohorts and were not confirmed in some retrospective studies using tumour tissue $[55,56]$. Regardless, mCRPC with biallelic $B R C A 2$ defects are vulnerable to therapies exploiting defective HRR, such as platinum chemotherapy or poly (ADP-ribose) polymerase (PARP) inhibitors [57-62]. Since a large minority of $\mathrm{mCRPC}$ have low levels of ctDNA, practitioners must understand the context of a 'negative' result when using liquid biopsies to screen for HRR defects. If there is no evidence for ctDNA in the sample, then the tumour may still carry somatic HRR alterations, and tissue testing should be pursued. Most DNA repair defects appear to be truncal to the metastatic lineage, so reflex testing of either archival primary tissue or metastatic biopsy is appropriate $[18,63]$.

In PCa, other HRR genes such as BRCA1, $P A L B 2$, and $R A D 51$ are altered at frequencies below $1 \%[24,48,64]$. It is plausible that affected mCRPC tumours are vulnerable to PARP inhibitors, but to date no clinical trials have been powered to address this question. Conversely, ATM and CDK12 mutations are prevalent in $\mathrm{mCRPC}$, but their direct association with HRR is tenuous, with unique implications for PARP inhibitor response. CDK12 mutations are linked to a distinct tandem duplicator phenotype and poor prognosis with standard of care treatments [65-69]. In CDK12-mutant mCRPC, PARP inhibitor response rates have been low, regardless of patient selection via liquid or tissue biopsy. The frequent tandem duplications in CDK12-mutant tumours may result in an elevated neoantigen burden and sensitivity to immune checkpoint blockade, but this hypothesis is untested in clinical trials $[67,68]$. Conversely, ATM mutations have not been linked to a genomic phenotype. The prognosis of mCRPC with ATM mutations is unclear, but as with $C D K 12$, response rates to PARP inhibitors appear to be reduced in comparison to BRCA2 [62]. Currently, prospective plasma ctDNA sequencing is under evaluation in several phase II/III PARP inhibitor clinical trials in mCRPC, and upon regulatory approval is likely to be key for patient biomarker screening. The largest hurdle to be overcome for reliance on plasma ctDNA screening is the detection of BRCA2 biallelic deletions, which (unlike ATM and CDK12) are recurrent in mCRPC.

DNA mismatch repair defects are present in 3\% to $5 \%$ of mCRPC $[18,24]$. MSH2 and MSH6 alterations predominate and can take the form of complex structural rearrangements, thus complicating detection strategies [70]. Like HRR-deficient tumours, those with mismatch repair deficiency (MMRd) display distinctive mutational signatures, including hypermutation (C $>$ T transitions, particularly in the NCG trinucleotide context) and microsatellite instability. MMRd signatures can be detected in plasma ctDNA from patients with mCRPC $[17,18,71]$. Although high tumour mutational burden is not exclusive to MMRd etiology, in PCa there are no other common causes of hypermutation, and assays assessing mutational burden in ctDNA can be used [18]. Patients with MMRd mCRPC may respond to immune checkpoint inhibitors [72].

In the context of DNA repair defects and PARP inhibitors, serial sampling can enable resistance mechanism identification. BRCA2 reversion mutations can be detected in plasma ctDNA at clinical progression on platinum chemotherapy or PARP inhibitors $[13,40,73-77]$. Plasma cfDNA sequencing identifies a greater diversity of BRCA2 reversion mutations than biopsy of a single metastatic site [73]. It is plausible that regular plasma cfDNA screening could detect emerging BRCA2 reversion mutations prior to clinical progression, offering opportunities for earlier interventions.

\section{AR Mutations, Amplifications, and Genomic Structural Rearrangements}

Missense mutations in the $A R$ ligand-binding domain can alter ligand affinity and drive therapy resistance and/or indicate potential vulnerabilities. Overall, $A R$ mutations are found in $10 \%$ of ctDNA-positive mCRPC, but few point mutations are widely recurrent, principally L702H, W742L/C, H875Y and T878A [23,32,33,39,78]. $A R$ W742L/C mutations are a resistance mechanism to bicalutamide and are frequently identified in the plasma ctDNA of bicalutamide treated patients. Nextgeneration AR-targeted therapies have activity against $A R \mathrm{~W} 742 \mathrm{~L} / \mathrm{C}$, meaning that its detection via liquid biopsy may predict durable responses to enzalutamide and abiraterone $[23,39]$. AR T878A and $\mathrm{L} 702 \mathrm{H}$ tend to arise after therapy, permitting agonism of the AR by 
progesterones and glucocorticoids, respectively $[32,33]$. While detection of these alterations in plasma cfDNA is linked to poor outcomes, switching to different ARtargeted therapies or steroid regimens may be effective in some scenarios [79].

$A R$ copy number gain is the most frequent category of $A R$ gene alteration in $\mathrm{mCRPC}$, enabling tumours to adapt to low androgen levels during treatment [15]. $A R$ copy gain in plasma cfDNA has been associated with shorter progression-free survival and overall survival in mCRPC patients treated with AR-targeted therapy $[23,32,37,39,80-84]$. However, measuring $A R$ copy gain in plasma cfDNA is complicated by variability in ctDNA abundance between mCRPC patients. Resolving a single extra $A R$ gene copy requires a ctDNA fraction of approximately $20 \%$, whereas the signal from $8 A R$ copies can be detected at a ctDNA fraction of only $5 \%$ [23]. In reality, $A R$ gain is not binary, but rather a continuous variable capturing increasing $A R$ copies, and an $A R$ copy dose-effect relationship with patient prognosis is plausible in the advanced-disease setting [23]. Interestingly, plasma $A R$ copy gain ris not associated with poor outcomes in MCRPC patients treated with taxane chemotherapy, suggesting an opportunity for a predictive biomarker [85].

$A R$ copy gain acquisition requires a series of structural rearrangements affecting the $A R$ locus. Genomic breakpoints falling within the $A R$ itself are termed $A R$-GSRs (genomic structural rearrangements). Some $A R$-GSRs result in a transcript coding for a truncated ligand-binding domain, similar in concept to the splice variant $A R v 7$ but usually distinct in nucleic acid sequence $[86,87]$. While the downstream consequences are challenging to predict from DNA breakpoints alone, in vitro studies have suggested that select $A R$-GSRs give rise to constitutively active $\mathrm{AR}$ proteins and drive therapy-resistant phenotypes. AR-GSRs can be detected via ctDNA sequencing of $A R$ introns, and are linked to primary resistance to AR-targeted therapies $[23,88]$. The presence of $A R$-GSRs is positively correlated with $A R$ copy number $[86,89]$, and $A R$-GSRs appear to be more abundant in patients with late-stage disease than initial mCRPC progression [17].

\section{Other Common Genomic Alterations as Potential Biomarkers}

The tumour suppressor TP53 is altered in over 50\% of mCRPC $[12,48]$. TP53 alterations detected in plasma cfDNA are linked to worse overall survival and poor response to AR-targeted therapy $[23,79,89]$, independent of ctDNA fraction and clinical prognostic factors [23]. PCa lacking the tumour suppressor triumvirate of $T P 53, R B 1$, and $P T E N$ are generally clinically aggressive and primed for lineage plasticity and rapid adaptation to therapy-induced bottlenecks $[90,91]$. Potentially aggressive disease variants can be identified at an early stage through the detection of TP53, RB1, and PTEN alterations in ctDNA [37]. Conversely, SPOP mutations appear to be a good prognostic factor when identified in either ctDNA or tissue $[23,92]$.

PTEN deletion is the most common PI3K pathway alteration in mCRPC [48]. Other alterations affect this pathway, including activating missense mutations in $A K T 1$ and PIK3CA in 6\% of patients [93]. Tumours with PI3K alterations may be reliant on PI3K signalling for survival and therefore represent a therapeutic vulnerability. PTEN deletion appears to be a biomarker for selecting patients most likely to respond to PI3K pathway inhibition [94]. Consequently, the pan-Akt inhibitor ipatasertib is under evaluation in a phase III clinical trial in mCRPC patients with PTEN defects (NCT03072238). It is unclear whether other PI3K signalling pathway alterations will be relevant for ipatasertib response if the drug is approved. However, a recent ctDNA-based study suggested that patients with somatic truncal hotspot mutations in $A K T 1$ or PIK3CA are reliant on the pathway and may have strong responses to ipatasertib [93].

\section{Non-Genomic Information Available in Cell-Free DNA}

In addition to genomic alteration status, ctDNA profiling can inform on aspects of the epigenome. Nucleosomes protect cfDNA from degradation by circulating nucleases, and their positioning can be inferred from whole-genome mapping of cfDNA fragments. Patterns of nucleosome spacing indicate tissue of origin and were crucial in demonstrating that plasma cfDNA is largely derived from hematopoietic cells [1]. In addition, plasma cfDNA fragmentation patterns vary between cancer patients and healthy individuals, indicating diagnostic potential [2]. Lastly, the non-random fragmentation pattern of ctDNA means that even transcription factor activity and gene expression can be inferred from wholegenome cfDNA sequencing $[95,96]$.

Epigenetic marks, such as cytosine methylation, are tissue- and cancer-specific features present on cfDNA/ ctDNA fragments $[97,98]$. Therefore, tissue of origin can also be predicted through methylation profiling of cfDNA $[7,99]$. Importantly, the number of cell typespecific methylation marks in a tumour cell vastly outnumbers the somatic mutation count. Therefore, plasma cfDNA methylation assays have potential for greater ctDNA detection sensitivity than assays reliant on capturing somatic mutations, especially in the context of early cancer diagnosis $[97,98]$. Detection of prostate lineage methylation marks on cfDNA can provide an accurate measure of ctDNA fraction and 
even resolve patients with $A R$ copy gain [100]. Finally, a recent study demonstrated that in a subset of mCRPC patients with high ctDNA fractions, simultaneous whole-exome sequencing and whole-genome bisulfite sequencing can identify neuroendocrine PCa [25].

\section{Future Directions}

True clinical translation of recent ctDNA correlative research will require prospective clinical trials. In the context of DNA repair and PARP inhibition, cfDNA analysis is part of patient screening protocols in several ongoing phase II/III trials [61]. The innovative ProBio clinical trial (NCT03903835) is an outcome-adaptive, multi-arm, platform trial testing the utility of liquid biopsies to tailor treatment decisions in MCRPC [101]. The initial arms in this trial will test prognostic and predictive biomarkers for many of the current standard of care therapies such as abiraterone and cabazitaxel. PC-BETS (NCT03385655; also known as IND234) is another multi-arm umbrella trial, but set in a later stage than ProBio, testing investigative agents such as adavosertib, darolutamide, palbociclib, and ipatasertib. CtDNA fraction as a potential biomarker is also being prospectively tested in the phase II PROTRACT trial (NCT04015622). In PROTRACT, mCRPC patients who have progressed on abiraterone are randomized

\section{References}

1. Snyder MW, Kircher M, Hill AJ, Daza RM, Shendure J. Cell-free DNA comprises an in vivo nucleosome footprint that informs its tissues-of-origin. Cell. 2016 Jan 14;164(1-2):57-68. doi. org/10.1016/j.cell.2015.11.050

2. Cristiano S, Leal A, Phallen J, et al. Genome-wide cell-free DNA fragmentation in patients with cancer. Nature. 2019 Jun;570(7761):385-9. doi.org/10.1038/s41586-019-1272-6

3. Meddeb R, Dache ZAA, Thezenas $S$, et al. Quantifying circulating cell-free DNA in humans. Sci Rep. 2019 Mar 26;9(1):5220. doi. org/10.1038/s41598-019-41593-4

4. Mouliere F, Chandrananda D, Piskorz AM, et al. Enhanced detection of circulating tumor DNA by fragment size analysis. Sci Trans/ Med. 2018 Nov 7;10(466). doi.org/10.1126/scitranslmed.aat4921

5. Greytak SR, Engel KB, Parpart-Li S, et al. Harmonizing cell-free DNA collection and processing practices through evidencebased guidance. Clin Cancer Res. 2020 Jul 1;26(13):3104-9. doi. org/10.1158/1078-0432.CCR-19-3015

6. Cheng AP, Burnham P, Lee JR, et al. A cell-free DNA metagenomic sequencing assay that integrates the host injury response to infection. Proc Natl Acad Sci U S A. 2019 Sep 10;116(37):18738-44. doi.org/10.1073/pnas.1906320116

7. Moss J, Magenheim J, Neiman D, et al. Comprehensive human cell-type methylation atlas reveals origins of circulating cell-free DNA in health and disease. Nat Commun. 2018 Nov 29;9(1):5068. doi.org/10.1038/s41467-018-07466-6 to physician's choice of enzalutamide or docetaxel, or a biomarker-driven stratification based on pre-treatment ctDNA fraction. The results from these and similar trials will be crucial for moving cfDNA profiling towards routine clinical use in $\mathrm{mCRPC}$.

Beyond prospective validation, there are several other hurdles that must be overcome before ctDNA analysis will become part of standard clinical care. With the sensitivity of current technology, liquid biopsy will remain uninformative for somatic alterations in those patients with minuscule plasma ctDNA levels, meaning that tumour tissue testing must remain part of the molecular diagnostics paradigm. Teaching practitioners how to interpret liquid biopsy results will be key to development of workflows that optimally use both ctDNA and tissue testing at appropriate times during $\mathrm{PCa}$ progression. Furthermore, the current range of commercially available ctDNA tests are not tailored for $\mathrm{PCa}$, and most of the translational studies described in this review leveraged bespoke research-based sequencing approaches that cannot be implemented in a large clinical system. Therefore, the development of clinical-grade liquid biopsy assays that are specific for the unique genomic features of PCa will help speed the uptake of ctDNA testing in the real world.

8. Diehl F, Schmidt K, Choti MA, et al. Circulating mutant DNA to assess tumor dynamics. Nat Med. 2008 Sep;14(9):985-90. doi. org/10.1038/nm.1789

9. Bettegowda C, Sausen M, Leary RJ, et al. Detection of circulating tumor DNA in early- and late-stage human malignancies. Sci Trans/ Med. 2014 Feb 19;6(224):224ra24. doi.org/10.1126/ scitranslmed.3007094

10. Cescon DW, Bratman SV, Chan SM, Siu LL. Circulating tumor DNA and liquid biopsy in oncology. Nat Cancer. 2020;1:276-90. doi. org/10.1038/s43018-020-0043-5

11. Cancer Genome Atlas Research Network. The molecular taxonomy of primary prostate cancer. Cell. 2015 Nov 5;163(4):1011-25. doi. org/10.1016/j.cell.2015.10.025

12. Armenia J, Wankowicz SAM, Liu D, et al. The long tail of oncogenic drivers in prostate cancer. Nat Genet. 2018 May;50(5):645-51. doi. org/10.1038/s41588-018-0078-z

13. Taavitsainen S, Annala M, Ledet E, et al. Evaluation of commercial circulating tumor DNA test in metastatic prostate cancer. JCO Precis Oncol. 2019:1-9. doi: 10.1200/P0.19.00014

14. Maia MC, Salgia M, Pal SK. Harnessing cell-free DNA: plasma circulating tumour DNA for liquid biopsy in genitourinary cancers. Nat Rev Urol. 2020;17:271-291. doi.org/10.1038/s41585-020-0297-9

15. Quigley DA, Dang HX, Zhao SG, et al. Genomic hallmarks and structural variation in metastatic prostate cancer. Cell. 2018 0ct 18;175(3):889. doi.org/10.1016/j.cell.2018.10.019 
16. Fraser M, Sabelnykova VY, Yamaguchi TN, et al. Genomic hallmarks of localized, non-indolent prostate cancer. Nature. 2017 Jan 19;541(7637):359-64. doi.org/10.1038/nature20788

17. Mayrhofer M, De Laere B, Whitington T, et al. Cell-free DNA profiling of metastatic prostate cancer reveals microsatellite instability, structural rearrangements and clonal hematopoiesis. Genome Med. 2018 Nov 21;10(1):85. doi.org/10.1186/s13073-018-0595-5

18. Ritch E, Fu SYF, Herberts C, et al. Identification of hypermutation and defective mismatch repair in ctDNA from metastatic prostate cancer. Clin Cancer Res. 2020 Mar 1;26(5):1114-25. doi. org/10.1158/1078-0432.CCR-19-1623

19. Nava Rodrigues D, Casiraghi N, Romanel A, et al. RB1 Heterogeneity in advanced metastatic castration-resistant prostate cancer. Clin Cancer Res. 2019 Jan 15;25(2):687-97. doi.org/10.1158/10780432.CCR-18-2068

20. Parolia A, Cieslik M, Chu S-C, et al. Distinct structural classes of activating FOXA1 alterations in advanced prostate cancer. Nature. 2019 Jul;571(7765):413-8. doi.org/10.1038/s41586-019-1347-4

21. Newman AM, Lovejoy AF, Klass DM, et al. Integrated digital error suppression for improved detection of circulating tumor DNA. Nat Biotechnol. 2016 May;34(5):547-55. doi.org/10.1038/nbt.3520

22. Phallen J, Sausen M, Adleff V, et al. Direct detection of early-stage cancers using circulating tumor DNA. Sci Trans/ Med. 2017 Aug 16;9(403). doi.org/10.1126/scitransImed.aan2415

23. Annala M, Vandekerkhove G, Khalaf D, et al. Circulating tumor DNA genomics correlate with resistance to abiraterone and enzalutamide in prostate cancer. Cancer Discov. 2018 Apr;8(4):444-57. doi. org/10.1158/2159-8290.CD-17-0937

24. Abida W, Cyrta J, Heller G, et al. Genomic correlates of clinical outcome in advanced prostate cancer. Proc Natl Acad Sci U S A. 2019 Jun 4;116(23):11428-36. doi.org/10.1073/pnas.1902651116

25. Beltran H, Romanel A, Conteduca V, et al. Circulating tumor DNA profile recognizes transformation to castration-resistant neuroendocrine prostate cancer. J Clin Invest. 2020 Apr 1;130(4):1653-68. doi.org/10.1172/JCI131041

26. Choudhury $A D$, Werner $L$, Francini $E$, et al. Tumor fraction in cellfree DNA as a biomarker in prostate cancer. JCl Insight. $2018 \mathrm{Nov}$ 2;3(21). doi.org/10.1172/jci.insight.122109

27. Adalsteinsson VA, Ha G, Freeman SS, et al. Scalable wholeexome sequencing of cell-free DNA reveals high concordance with metastatic tumors. Nat Commun. 2017 Nov 6;8(1):1324. doi. org/10.1038/s41467-017-00965-y

28. Giri VN, Knudsen KE, Kelly WK, et al. Role of genetic testing for inherited prostate cancer risk: Philadelphia Prostate Cancer Consensus Conference 2017. J Clin Oncol. 2018 Feb 1;36(4):414-24. doi.org/10.1200/JC0.2017.74.1173

29. Razavi P, Li BT, Brown DN, et al. High-intensity sequencing reveals the sources of plasma circulating cell-free DNA variants. Nat Med. 2019 Dec;25(12):1928-37. doi.org/10.1038/s41591-019-0652-7

30. Stetson D, Ahmed A, Xu X, et al. Orthogonal comparison of four plasma NGS tests with tumor suggests technical factors are a major source of assay discordance. JCO Precis Oncol. 2019:1-9. doi.org/10.1200/po.18.00191
31. Torga G, Pienta KJ. Patient-paired sample congruence between 2 commercial liquid biopsy tests. JAMA Oncol. 2018 Jun 1;4(6):86870. doi.org/10.1001/jamaoncol.2017.4027

32. Romanel A, Gasi Tandefelt D, Conteduca V, et al. Plasma AR and abiraterone-resistant prostate cancer. Sci Trans/ Med. 2015 Nov 4;7(312):312re10. doi.org/10.1126/scitransImed.aac9511

33. Carreira S, Romanel A, Goodall J, et al. Tumor clone dynamics in lethal prostate cancer. Sci Trans/ Med. 2014 Sep 17;6(254):254ra125. doi.org/10.1126/scitransImed.3009448

34. Schweizer MT, Gulati R, Beightol M, et al. Clinical determinants for successful circulating tumor DNA analysis in prostate cancer. Prostate. 2019 May;79(7):701-8.doi.org/10.1002/pros.23778

35. De Laere B, Oeyen S, Mayrhofer M, et al. TP53 outperforms other androgen receptor biomarkers to predict abiraterone or enzalutamide outcome in metastatic castration-resistant prostate cancer. Clin Cancer Res. 2019 Mar 15;25(6):1766-73. doi.org/10.1158/10780432.CCR-18-1943

36. Mehra N, Dolling D, Sumanasuriya S, et al. Plasma cell-free DNA concentration and outcomes from taxane therapy in metastatic castration-resistant prostate cancer from two phase III trials (FIRSTANA and PROSELICA). Eur Urol. 2018 Sep;74(3):283-91.doi. org/10.1016/j.eururo.2018.02.013

37. Kohli M, Tan W, Zheng T, et al. Clinical and genomic insights into circulating tumor DNA-based alterations across the spectrum of metastatic hormone-sensitive and castrate-resistant prostate cancer. EBioMedicine. 2020 Apr 5;54:102728. doi.org/10.1016/j. ebiom. 2020.102728

38. Vandekerkhove G, Struss WJ, Annala M, et al. Circulating tumor DNA abundance and potential utility in de novo metastatic prostate cancer. Eur Urol. 2019 Apr;75(4):667-75. doi.org/10.1016/j. eururo.2018.12.042

39. Wyatt AW, Azad AA, Volik SV, et al. Genomic alterations in cell-free DNA and enzalutamide resistance in castration-resistant prostate cancer. JAMA Oncol. 2016 Dec 1;2(12):1598-606. doi.org/10.1001/ jamaoncol.2016.0494

40. Goodall J, Mateo J, Yuan W, et al. Circulating cell-free DNA to guide prostate cancer treatment with PARP inhibition. Cancer Discov. 2017 Sep;7(9):1006-17. doi.org/10.1158/2159-8290.CD-17-0261

41. Chi KN, Taavitsainen S, Iqbal N, et al. Updated results from a randomized phase II study of cabazitaxel (CAB) versus abiraterone (ABI) or enzalutamide (ENZ) in poor prognosis metastatic CRPC. J Clin Oncol. 2019;37:5003-5003. doi.org/10.1200/ jco.2019.37.15_suppl.5003

42. Wyatt AW, Annala M, Aggarwal R, et al. Concordance of circulating tumor DNA and matched metastatic tissue biopsy in prostate cancer. J Natl Cancer Inst. 2017 Dec 1;109(12). doi.org/10.1093/ jnci/djx118

43. Hovelson DH, Liu C-J, Wang Y, et al. Rapid, ultra low coverage copy number profiling of cell-free DNA as a precision oncology screening strategy. Oncotarget. 2017 0ct 27;8(52):89848-66. doi. org/10.18632/oncotarget.21163

44. Gundem G, Van Loo P, Kremeyer B, et al. The evolutionary history of lethal metastatic prostate cancer. Nature. $2015 \mathrm{Apr}$ 16;520(7547):353-7. doi.org/10.1038/nature14347 
45. Kumar A, Coleman I, Morrissey C, et al. Substantial interindividual and limited intraindividual genomic diversity among tumors from men with metastatic prostate cancer. Nat Med. 2016 Apr;22(4):36978. doi.org/10.1038/nm.4053

46. Mateo J, Boysen G, Barbieri CE, Bryant HE, Castro E, Nelson $P S$, et al. DNA repair in prostate cancer: biology and clinical implications. Eur Urol. 2017 Mar;71(3):417-25. doi.org/10.1016/j. eururo.2016.08.037

47. Warner EW, Yip SM, Chi KN, Wyatt AW. DNA repair defects in prostate cancer: impact for screening, prognostication and treatment. BJU Int. 2019 May;123(5):769-76. doi.org/10.1111/ bju. 14576

48. Robinson D, Van Allen EM, Wu Y-M, et al. Integrative clinical genomics of advanced prostate cancer. Cell. 2015 Jul 16;162(2):454. doi.org/10.1016/j.cell.2015.06.053

49. Beltran H, Yelensky R, Frampton GM, et al. Targeted next-generation sequencing of advanced prostate cancer identifies potential therapeutic targets and disease heterogeneity. Eur Urol. 2013 May;63(5):920-6. doi.org/10.1016/j.eururo.2012.08.053

50. Lord CJ, Ashworth A. PARP inhibitors: Synthetic lethality in the clinic. Science. 2017 Mar 17;355(6330):1152-8. doi.org/10.1126/ science.aam7344

51. Annala M, Struss WJ, Warner EW, et al. Treatment outcomes and tumor loss of heterozygosity in germline DNA repair-deficient Prostate Cancer. Eur Urol. 2017 Jul;72(1):34-42. doi.org/10.1016/j. eururo.2017.02.023

52. Sztupinszki Z, Diossy M, Krzystanek M, et al. Detection of molecular signatures of homologous recombination deficiency in prostate cancer with or without BRCA1/2 mutations. Clin Cancer Res. 2020 Feb 18. doi.org/10.1158/1078-0432.CCR-19-2135

53. Jonsson $P$, Bandlamudi $C$, Cheng ML, et al. Tumour lineage shapes BRCA-mediated phenotypes. Nature. 2019 Jul;571(7766):576-9. doi.org/10.1038/s41586-019-1382-1

54. Castro E, Romero-Laorden N, Del Pozo A, et al. PROREPAIR-B: A prospective cohort study of the impact of germline DNA repair mutations on the outcomes of patients with metastatic castrationresistant prostate cancer. J Clin Oncol. 2019 Feb 20;37(6):490-503. doi.org/10.1200/JC0.18.00358

55. Mateo J, Cheng HH, Beltran H, et al. Clinical outcome of prostate cancer patients with germline DNA repair mutations: retrospective analysis from an international study. Eur Urol. 2018 May;73(5):68793. doi.org/10.1016/j.eururo.2018.01.010

56. Hussain M, Daignault-Newton S, Twardowski PW, et al. Targeting androgen receptor and DNA repair in metastatic castration-resistant prostate cancer: results from NCl 9012. J Clin Oncol. 2018 Apr 1;36(10):991-9. doi.org/10.1200/JC0.2017.75.7310

57. Marshall CH, Sokolova AO, McNatty AL, et al. Differential response to olaparib treatment among men with metastatic castrationresistant prostate cancer harboring BRCA1 or BRCA2 versus ATM mutations. Eur Urol. 2019 Oct;76(4):452-8. doi.org/10.1016/j. eururo.2019.02.002
58. Mateo J, Carreira S, Sandhu S, et al. DNA-repair defects and olaparib in metastatic prostate cancer. N Engl J Med. 2015 Oct 29;373(18):1697-708. doi.org/10.1056/NEJMoa1506859

59. Pomerantz MM, Spisák S, Jia L, et al. The association between germline BRCA2 variants and sensitivity to platinum-based chemotherapy among men with metastatic prostate cancer. Cancer. 2017 Sep 15;123(18):3532-9. doi.org/10.1002/cncr.30808

60. de Bono J, Mateo J, Fizazi K, et al. Olaparib for metastatic castration-resistant prostate cancer. N Engl J Med. 2020 April 28; doi.org/10.1056/NEJMoa1911440

61. Ratta R, Guida A, Scotté F, et al. PARP inhibitors as a new therapeutic option in metastatic prostate cancer: a systematic review. Prostate Cancer Prostatic Dis. 2020 May 4; doi.org/10.1038/ s41391-020-0233-3

62. Mateo J, Porta N, Bianchini D, et al. Olaparib in patients with metastatic castration-resistant prostate cancer with DNA repair gene aberrations (TOPARP-B): a multicentre, open-label, randomised, phase 2 trial. Lancet Oncol. 2020 Jan;21(1):162-74. doi.org/10.1016/S1470-2045(19)30684-9

63. Mateo J, Seed G, Bertan C, et al. Genomics of lethal prostate cancer at diagnosis and castration resistance. J Clin Invest. 2020 Apr 1;130(4):1743-51. doi.org/10.1172/JCI132031

64. Pritchard CC, Mateo J, Walsh MF, et al. Inherited DNA-repair gene mutations in men with metastatic prostate cancer. N Engl J Med. 2016 Aug 4;375(5):443-53. doi.org/10.1056/NEJMoa1603144

65. Nguyen B, Mota JM, Nandakumar S, et al. Pan-cancer analysis of CDK12 alterations identifies a subset of prostate cancers with distinct genomic and clinical characteristics. Eur Urol. 2020 Apr 19; doi.org/10.1016/j.eururo.2020.03.024

66. Reimers MA, Yip SM, Zhang L, et al. Clinical outcomes in cyclindependent kinase 12 mutant advanced prostate cancer. Eur Urol. 2020 Mar;77(3):333-41. doi.org/10.1016/j.eururo.2019.09.036

67. Antonarakis ES, Isaacsson Velho P, Fu W, et al. CDK12-altered prostate cancer: clinical features and therapeutic outcomes to standard systemic therapies, poly (ADP-Ribose) polymerase inhibitors, and PD-1 inhibitors. JCO Precis Oncol. 2020 Apr;(4):37081. doi/10.1200/P0.19.00399

68. Wu Y-M, Cieślik M, Lonigro RJ, et al. Inactivation of CDK12 delineates a distinct immunogenic class of advanced prostate cancer. Cell. 2018 Jun 14;173(7):1770-82.e14. doi.org/10.1016/j. cell.2018.04.034

69. Schweizer MT, Ha G, Gulati R, et al. CDK12-mutated prostate cancer: clinical outcomes with standard therapies and immune checkpoint blockade. JCO Precis Oncol. 2020:382-92. doi. org/10.1200/po.19.00383

70. Pritchard CC, Morrissey C, Kumar A, et al. Complex MSH2 and MSH6 mutations in hypermutated microsatellite unstable advanced prostate cancer. Nat Commun. 2014 Sep 25;5:4988. doi.org/10.1038/ ncomms5988 
71. Manogue C, Cotogno P, Ledet E, Lewis B, Wyatt AW, Sartor 0 . Biomarkers for programmed death-1 inhibition in prostate cancer. Oncologist. 2019 Apr;24(4):444-8. doi.org/10.1634/ theoncologist.2018-0546

72. Antonarakis ES, Shaukat F, Isaacsson Velho P, et al. Clinical features and therapeutic outcomes in men with advanced prostate cancer and DNA mismatch repair gene mutations. Eur Urol. 2019 Mar;75(3):378-82. doi.org/10.1016/j.eururo.2018.10.009

73. Quigley D, Alumkal JJ, Wyatt AW, et al. Analysis of circulating cell-free DNA identifies multiclonal heterogeneity of reversion mutations associated with resistance to PARP inhibitors. Cancer Discov. 2017 Sep;7(9):999-1005. doi.org/10.1158/2159-8290. CD-17-0146

74. Vidula N, Rich TA, Sartor O, et al. Routine plasma-based genotyping to comprehensively detect germline, somatic, and reversion mutations among patients with advanced solid tumors. Clin Cancer Res. 2020 Feb 7; doi.org/10.1158/1078-0432.CCR-19-2933

75. Cheng HH, Salipante SJ, Nelson PS, Montgomery B, Pritchard CC. Polyclonal BRCA2 reversion mutations detected in circulating tumor DNA after platinum chemotherapy in a patient with metastatic prostate cancer. JCO Precis Oncol. 2018. p. 1-5. doi.org/10.1200/ po.17.00169

76. Simmons AD, Nguyen M, Pintus E. Polyclonal BRCA2 mutations following carboplatin treatment confer resistance to the PARP inhibitor rucaparib in a patient with mCRPC: a case report. $B M C$ Cancer. 2020;20: doi.org/10.1186/s12885-020-6657-2

77. Carneiro BA, Collier KA, Nagy RJ, et al. Acquired resistance to poly (ADP-ribose) polymerase inhibitor olaparib in BRCA2-associated prostate cancer resulting from biallelic BRCA2 reversion mutations restores both germline and somatic loss-of-function mutations. JCO Precis Oncol. 2018:1-8. doi.org/10.1200/po.17.00176

78. Ledet EM, Lilly MB, Sonpavde G, et al. Comprehensive analysis of $A R$ alterations in circulating tumor DNA from patients with advanced prostate cancer. Oncologist. 2020 Apr;25(4):327-33. doi.org/10.1634/theoncologist.2019-0115

79. Torquato S, Pallavajjala A, Goldstein A, et al. Genetic alterations detected in cell-free DNA are associated with enzalutamide and abiraterone resistance in castration-resistant prostate cancer. JCO Precis Oncol. 2019 Apr 3;3. doi.org/10.1200/P0.18.00227

80. Conteduca V, Wetterskog D, Sharabiani MTA, et al. Androgen receptor gene status in plasma DNA associates with worse outcome on enzalutamide or abiraterone for castration-resistant prostate cancer: a multi-institution correlative biomarker study. Ann Oncol. 2017 Jul 1;28(7):1508-16. doi.org/10.1093/annonc/mdx155

81. Azad AA, Volik SV, Wyatt AW, et al. Androgen receptor gene aberrations in circulating cell-free DNA: biomarkers of therapeutic resistance in castration-resistant prostate cancer. Clin Cancer Res. 2015 May 15;21(10):2315-24. doi.org/10.1158/1078-0432. CCR-14-2666
82. Salvi S, Casadio V, Conteduca V, et al. Circulating cell-free AR and CYP17A1 copy number variations may associate with outcome of metastatic castration-resistant prostate cancer patients treated with abiraterone. Br J Cancer. 2015 May 12;112(10):1717-24. doi. org/10.1038/bjc.2015.128

83. Du M, Tian Y, Tan W, et al. Plasma cell-free DNA-based predictors of response to abiraterone acetate/prednisone and prognostic factors in metastatic castration-resistant prostate cancer. Prostate Cancer Prostatic Dis. 2020 Mar 18; doi.org/10.1038/s41391-020-0224-4

84. Jayaram A, Wingate A, Wetterskog D, et al. Plasma androgen receptor copy number status at emergence of metastatic castrationresistant prostate cancer: a pooled multicohort analysis. JCO Precis Oncol. 2019:1-13. doi.org/10.1200/po.19.00123

85. Conteduca V, Jayaram A, Romero-Laorden N, et al. Plasma androgen receptor and docetaxel for metastatic castration-resistant prostate cancer. Eur Urol. 2019 Mar;75(3):368-73. doi.org/10.1016/j. eururo.2018.09.049

86. Li Y, Yang R, Henzler CM, et al. Diverse gene rearrangements mediate resistance to androgen receptor inhibitors in metastatic prostate cancer. Clin Cancer Res. 2020 Apr 15;26(8):1965-76. http:// dx.doi.org/10.1158/1078-0432.CCR-19-3023

87. Henzler C, Li Y, Yang R, et al. Truncation and constitutive activation of the androgen receptor by diverse genomic rearrangements in prostate cancer. Nat Commun. 2016 Nov 29;7:13668. doi. org/10.1038/ncomms13668

88. De Laere B, van Dam P-J, Whitington T, et al. Comprehensive profiling of the androgen receptor in liquid biopsies from castrationresistant prostate cancer reveals novel intra-ar structural variation and splice variant expression patterns. Eur Urol. 2017 Aug;72(2):192-200. doi.org/10.1016/j.eururo.2017.01.011

89. De Laere B, Rajan P, Grönberg H, Dirix L, Lindberg J; CORE-ARVCTC and ProBIO Investigators. Androgen receptor burden and poor response to abiraterone or enzalutamide in TP53 wild-type metastatic castration-resistant prostate cancer. JAMA Oncol. 2019 Jul 1;5(7):1060-2.doi.org/10.1001/jamaoncol.2019.0869

90. Beltran H, Hruszkewycz A, Scher HI, et al. The role of lineage plasticity in prostate cancer therapy resistance. Clin Cancer Res. 2019 Dec 1;25(23):6916-24. doi.org/10.1158/1078-0432. CCR-19-1423

91. Hamid AA, Gray KP, Shaw G, et al. Compound genomic alterations of TP53, PTEN, and RB1 tumor suppressors in localized and metastatic prostate cancer. Eur Urol. 2019 Jul;76(1):89-97. doi.org/10.1016/j. eururo.2018.11.045

92. Boysen G, Rodrigues DN, Rescigno P, et al. SPOP-mutated/ CHD1-deleted lethal prostate cancer and abiraterone sensitivity. Clin Cancer Res. 2018 Nov 15;24(22):5585-93. doi.org/10.1158/10780432.CCR-18-0937

93. Herberts C, Murtha AJ, Fu S, et al. Activating AKT1 and PIK3CA mutations in metastatic castration-resistant prostate cancer. Eur Urol. 2020; In Press. 
94. de Bono JS, De Giorgi U, Rodrigues DN, et al. Randomized phase II study evaluating akt blockade with ipatasertib, in combination with abiraterone, in patients with metastatic prostate cancer with and without PTEN loss. Clin Cancer Res. 2019 Feb 1;25(3):928-36. doi. org/10.1158/1078-0432.CCR-18-0981

95. Ulz P, Perakis S, Zhou Q, et al. Inference of transcription factor binding from cell-free DNA enables tumor subtype prediction and early detection. Nat Commun. 2019 0ct 11;10(1):4666. doi. org/10.1038/s41467-019-12714-4

96. Ulz P, Thallinger GG, Auer M, et al. Inferring expressed genes by whole-genome sequencing of plasma DNA. Nat Genet. 2016 Oct;48(10):1273-8. doi.org/10.1038/ng.3648

97. Liu L, Toung JM, Jassowicz AF, et al. Targeted methylation sequencing of plasma cell-free DNA for cancer detection and classification. Ann Oncol. 2018 Jun 1;29(6):1445-53. doi. org/10.1093/annonc/mdy119
98. Shen SY, Singhania R, Fehringer G, et al. Sensitive tumour detection and classification using plasma cell-free DNA methylomes. Nature. 2018 Nov;563(7732):579-83. doi.org/10.1038/s41586-018-0703-0

99. Sun K, Jiang P, Chan KCA, et al. Plasma DNA tissue mapping by genome-wide methylation sequencing for noninvasive prenatal, cancer, and transplantation assessments. Proc Natl Acad Sci U S A. 2015 Oct 6;112(40):E5503-12. doi.org/10.1073/pnas.1508736112

100. Wu A, Cremaschi P, Wetterskog D, et al. Genome-wide plasma DNA methylation features of metastatic prostate cancer. J Clin Invest. 2020 Apr 1;130(4):1991-2000. doi.org/10.1172/JCI130887

101. Lindberg J, De Laere B, Crippa A, Eklund M, Grönberg H. ProBio: an outcome-adaptive, multi-arm, open-label, multiple assignment randomised controlled biomarker-driven trial in patients with metastatic castration-resistant prostate cancer. Ann Oncol. 2019;30:354. doi.org/10.1093/annonc/mdz248.053 